\title{
Spectrum of renal cortical necrosis in acute renal failure in Eastern India
}

\author{
Jai Prakash, K Tripathi, LK Pandey, Soma Sahai, Usha, PK Srivastava
}

\begin{abstract}
Summary
Renal cortical necrosis is an uncommon cause of acute renal failure. We report 23 cases of biopsy-proven renal cortical necrosis which constituted $6.3 \%(23 / 363)$ of all cases of acute renal failure studied over a period of seven years (1985-92). The patients were divided into two groups: obstetric and non-obstetric. Obstetric complications were responsible for renal cortical necrosis in $15(65.2 \%)$ patients while non-obstetric conditions accounted for the remaining eight $(34.8 \%)$ cases. The overall incidence of cortical necrosis in obstetric acute renal failure was $15 / 63(23.8 \%)$ patients, the incidence being nearly equal in early (20.5\%) and late (29\%) pregnancy. Postabortum renal failure was the sole cause of cortical necrosis in early pregnancy in the obstetric group. Haemolytic uraemic syndrome (three patients) and septicaemia (two patients) were the main cause of necrosis in the non-obstetric group. The cortical necrosis was diffuse and patchy in 17 and six patients, respectively. The disease had a fatal prognosis in $20(87 \%)$ patients; mortality was due to uraemic complications and infections in the majority of patients. The high frequency of post-abortum renal cortical necrosis in our patients is similar to the experience of other Indian workers.
\end{abstract}

Institute of Medical

Sciences, Banaras

Hindu University,

Varanasi-221005, India

Division of

Nephrology

J Prakash

Department of

Medicine

K Tripathi

Department of

Obstetrics and

Gynaecology

LK Pandey

S Sahai

Department of

Pathology

Usha

Indira Gandhi Institute of Medical

Sciences, Patna, India

PK Srivastava

Accepted 30 November 1994
Keywords: septic abortion, acute renal failure, cortical necrosis, India

\section{Introduction}

Acute bilateral renal cortical necrosis is a rare but potentially fatal variety of renal lesion. There is global necrosis of all of the elements in the involved area of the cortex, including blood vessels, glomeruli, and tubules. Irreversible loss of kidney function is the rule in complete cortical necrosis but is variable in the incomplete type where less than $50 \%$ of nephrons are necrotic. Renal cortical necrosis is an uncommon entity that accounts for only $2 \%$ of all causes of acute renal failure. ${ }^{1}$ Obstetric complications are the commonest $(50-70 \%$ ) cause of renal cortical necrosis 2,3 ; non-obstetric causes account for $20-30 \%$ of all cases of cortical necrosis and in these circumstances the incidence is higher in men than in women. ${ }^{4}$ The non-obstetric conditions leading to acute cortical necrosis are: extensive burns, pancreatitis, septicemia, snake bite, and diabetic ketoacidosis. ${ }^{5,6}$ Abruptio placentae, septic abortion, eclamptic toxaemia, post-partum haemorrhage and puerperal sepsis are the pregnancy-related situations responsible for renal cortical necrosis. ${ }^{1,7}$ The purpose of this paper is to study the causes, clinical course, and outcome of renal cortical necrosis in patients with acute renal failure.

\section{Methods}

Over a period of seven years (1985-92), 23 patients had biopsy-proven renal cortical necrosis out of a total of 363 cases of acute renal failure studied during this period. A complete history and physical examination findings with special emphasis on duration of oliguria/ anuria, hypotensive episodes, sepsis/septic shock and severity of renal failure were noted in each case. Laboratory investigations included estimation of urea, creatinine, total and differential leucocyte count, haemoglobin, serum uric acid, calcium, phosphorus, and alkaline phosphatase. Haematological and coagulation studies were conducted to rule out haemolysis and disseminated intravascular coagulation as and when required. Renal tissues were obtained by a percutaneous approach (necropsy in 15, biopsy in eight) in all patients and studied using light microscopy. Renal biopsy was done in eight patients with prolonged renal failure (oligoanuria lasting more than five weeks). The patients showing cortical necrosis on histology were selected for the present study. Based on the following criteria, patients were divided into two groups:

Complete cortical necrosis: essentially irreversible renal failure. Confluent global cortical destruction extending into the columns of Bertin. Thin rim of subcapsular and juxtamedullary tissue being preserved.

Patchy cortical necrosis: contiguous area of cortical necrosis up to one-third to one-half of entire cortical tissue. This form has potential for partial recovery of renal function.

\section{Results}

Of 363 patients with acute renal failure, 23 $(6.3 \%)$ had renal cortical necrosis. The patients fell into two groups: 15 obstetric $(65.2 \%)$, and eight non-obstetric $(34.8 \%)$. All patients were initially treated at peripheral centres (primary 
health centres or private practitioners) and were referred to us only after the development of acute renal failure with a duration of oligoanuria of 4-10 days. These patients had severe acute renal failure on admission with neuropsychiatric manifestations (11), metabolic acidosis (four), bleeding diathesis (two), and fluid overload (five). The severity of renal failure is shown in table 1 . All patients were treated with dialysis (peritoneal/ haemodialysis) in addition to supportive treatment using standard criteria until they improved or died. The obstetric complications leading to cortical necrosis are shown in table 2 . The cortical necrosis was observed in $15 / 63$ $(23.8 \%)$ patients with obstetric acute renal failure. The incidence of cortical necrosis in early and late pregnancy was nearly equal, $20.5 \%$ and $29 \%$, respectively. In the obstetric group, three patients $(20 \%)$ had patchy and 12 $(80 \%)$ had diffuse renal cortical necrosis There were eight cases (seven males; one female) in the non-obstetric group. The clinical features of the non-obstetric conditions leading to cortical necrosis are given in table 3. One patient survived with partial recovery of renal function in the obstetric group and two in the non-obstetric group.

The overall mortality was $20(87 \%)$. Death in 11 patients was attributed directly to uraemia, as the patients could not afford dialysis due to financial constraints. Uraemic complications leading to death were: profound azotemia (six), pulmonary oedema (three) and hyperkalaemia

Table 1 Severity of renal failure $(n=23)$

\begin{tabular}{lcr}
\hline Data & Range & Mean \\
\hline $\begin{array}{l}\text { Day of referral (days) } \\
\text { Peak pre-dialysis urea }\end{array}$ & $\begin{array}{c}4-10 \\
\text { (mg \%) }\end{array}$ & 5.3 \\
$\begin{array}{l}\text { Peak pre-dialysis creatinine } \\
\text { (mg \%) }\end{array}$ & $9-23.6$ & 168.2 \\
$\begin{array}{l}\text { Peak serum potassium } \\
\text { (mEq/l) }\end{array}$ & $4.2-7.8$ & 4.6 \\
\hline
\end{tabular}

Table 2 Obstetric causes of renal cortical necrosis $(n=15)$

\begin{tabular}{lll}
\hline Obstetric group & $n$ & $\%$ \\
\hline Post-abortum & 8 & 34.8 \\
Post-partum haemorrhage & 3 & 13.0 \\
Intra-uterine death & 2 & 8.7 \\
Puerperal sepsis & 2 & 8.7 \\
\hline
\end{tabular}

Table 3 Non-obstetric renal cortical necrosis $(n=8)$

\begin{tabular}{lccll}
\hline Conditions & Age & Sex & Type of necrosis & Results \\
\hline HUS & $18 \mathrm{~m}$ & $\mathrm{M}$ & Diffuse & Died \\
HUS & $8 \mathrm{yr}$ & $\mathrm{M}$ & Diffuse & Died \\
HUS & $6 \mathrm{~m}$ & $\mathrm{M}$ & Patchy & Survived \\
80\% burns, shock & $22 \mathrm{yr}$ & $\mathrm{F}$ & Diffuse & Died \\
Pneumonia sepsis, shock & $48 \mathrm{yr}$ & $\mathrm{M}$ & Patchy & Died \\
$\begin{array}{l}\text { Massive GI bleed, } \\
\text { hypotension }\end{array}$ & $36 \mathrm{yr}$ & $\mathrm{M}$ & Patchy & Died \\
Septic shock & $22 \mathrm{yr}$ & $\mathrm{M}$ & Diffuse & Died \\
Post-diarrhoeal & $18 \mathrm{yr}$ & $\mathrm{M}$ & Patchy & Survived \\
\hline
\end{tabular}

HUS = haemolytic uraemic syndrome; GI = gastrointestinal in (two) patients. Septicaemia was another major cause of mortality in eight $(34.8 \%)$ patients and one death was due to gastrointestinal haemorrhage. The majority of deaths occurred in the first two weeks (15/23) and necropsy specimens of renal tissue were obtained in all such cases for histological studies.

\section{Discussion}

Renal cortical necrosis is a rare entity that accounts for only $2 \%$ of all cases of acute renal failure. $^{1,3}$ Our study revealed $23(6.3 \%)$ patients had renal cortical necrosis among 363 patients with acute renal failure. The higher incidence of cortical necrosis in our patients with acute renal failure was possibly related to more frequent occurrence of cortical necrosis in post-abortal renal failure. Obstetric complications are responsible for $50-70 \%$ of cases of renal cortical necrosis in most reported

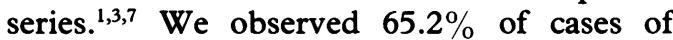
obstetric origin. Abruptio placentae is the most common obstetric situation associated with cortical necrosis. ${ }^{2,4}$ The peak incidence is in the last trimester of pregnancy and affects older, multiparous women. It is not clear why most victims of abruptio placentae who develop acute renal failure have acute tubular necrosis $(80 \%)$, whereas only a minority suffer from cortical necrosis.

Toxaemia of pregnancy, uterine haemorrhage, and puerperal sepsis are other conditions associated with cortical necrosis in late pregnany. ${ }^{7,8}$ Renal cortical necrosis is rare following septic abortion. ${ }^{9}$ The incidence of cortical necrosis in early pregnancy (post-abortum group) was $20.5 \%$ compared with $29.2 \%$ in late pregnancy. The high incidence of renal cortical necrosis in early pregnancy is the interesting feature of our study. Similar observations have been reported in an earlier report from this country. ${ }^{10}$ Thus we observed two peak incidences of obstetric cortical necrosis; one following septic abortion and the other in late pregnancy, in contrast to the findings in developed countries. Cortical necrosis unrelated to pregnancy accounts for $20-30 \%$ of all cases, and in a non-obstetric setting a higher incidence is observed in males than in females. ${ }^{4}$ There was only one woman in eight cases of non-obstetric cortical necrosis in the present study. In most cases, a combination of shock (either hypovolemic or septic) and disseminated intravascular coagulopathy is usually present. Four patients out of eight in our study had hypotension and shock in association with burns, sepsis, and massive gastro-intestinal haemorrhage. Patchy cortical necrosis was reported in one patient following diarrhoea. This patient had a partial recovery of renal function and is surviving without the need for dialysis. The anuria of cortical necrosis ( 0 to $50 \mathrm{ml} /$ day) is the striking feature in contrast to the 400 to $500 \mathrm{ml} /$ day output in acute tubular necrosis. ${ }^{11}$ Nineteen of 23 patients had absolute anuria and in four cases, 24-hour urinary output was less than $100 \mathrm{ml}$. The duration of anuria varied from 4-14 weeks. Evidence of 
disseminated intravascular coagulopathy in the form of thrombocytopenia, hypofibrogenemia, and prolonged prothrombin time was seen in seven and three patients in early and late pregnancy, respectively. The clinical course and outcome of patients with cortical necrosis can be classified into five broad groups (see box). Twenty ( $87 \%$ ) patients died during the acute phase in our study. The various causes of mortality were severe uraemia (six), pulmonary oedema (three), hyperkalaemia (two), gastrointestinal haemorrhage (one) and septicaemia (eight) patients. Thus, the majority of deaths which occurred in the acute phase were due to septicaemia and uraemia in patients who could not afford dialysis. However, the prognosis and survival of patients with cortical necrosis has improved markedly in developed countries due to the availability of renal replacement therpy. ${ }^{12,13}$ Three patients had a partial recovery of renal function and are surviving without dialysis support at six months follow up. They had prolonged oliguria (more than 35 days) and biopsy revealed patchy cortical necrosis. In certain patients, there may be a slow rise in creatinine clearance and a gradual gain in renal function over one to two years, so that the glomerular filtration rate may reach a final plateau level of approximately 20 to $25 \mathrm{ml} /$ min. ${ }^{14,15}$ It is assumed that juxtamedullary glomeruli (which comprise 15 to $20 \%$ of the total) escape destruction, even in complete cortical necrosis and that early functional return is due to recovery of these nephron segments. A significant number of patients may develop deterioration in renal function several years (1 to 10 years) after cortical necrosis. ${ }^{3}$ Factors causing this late functional downturn are not clear but include pyelonephritis, hypertension, and shrinkage of the kidneys due to progressive fibrosis and/or calcification. ${ }^{16,17}$

Acute bilateral cortical necrosis is an uncommon disease at the extreme end of the spectrum. Its incidence and severity has declined in obstetric practice in developed nations. In contrast to western countries renal cortical necrosis is still high in obstetric acute renal

1 Grunfeld JP, Gaveval D, Bournerias F. Acute renal failure in pregnancy. Kidney Int 1980; 18: 179-91.

2 Matlin RA, Gay NF. Acute cortical necrosis: case report and review of the literature. Am $\mathcal{F}$ Med 1974; 56: 110-8.

3 Kleinknecht D, Grunfeld JP, Cia Gomez P, Moreau JF, Garcia-Torres R. Diagnostic procedures and long term progress in bilateral cortical necrosis. Kidney Int 1973; 4: 390.

4 Duff GL, More RH. Bilateral cortical necrosis of the kidneys. Am $\mathcal{F}$ Med Sci 1941; 201: 429.

5 Lauler DP, Schreiner GE. Bilateral renal cortical necrosis Lauler DP, Schreiner GE.

6 Walls J, Schorr WJ, Kerr DNS: Prolonged oliguria with survival in acute bilateral cortical necrosis. BMF 1968; 4 $220-2$

7 Chugh KS, Singhal PC, Sharma BK, et al. Acute renal failure of obstetric origin. Obstet Gynaecol 1976; 48: 642-6.

8 Prakash J, Tripathi K, Usha, Srivastava PK. Pregnancy related acute renal failure is still high in India XIth International Congress of Nephrology, Tokyo, Japan 1990: 15A (abstract).

9 Smith K, Meclure Brown JC, Shockman R, Wrong OM Acute renal failure of obstetric origin. Lancet 1965; 2: 351-4.

10 Chugh KS, Singhal PC, Kher VK, et al. Spectrum of acute cortical necrosis in Indian patients. Am $\mathcal{F}$ Med Sci 1983; 286:

\section{Cortical necrosis: clinical course}

- death in uraemia during the acute phase

- survival without dialysis

- late return to dialysis/transplantation

- survival only with chronic maintenance dialysis/transplantation

- late resumption of sufficient renal function to become dialysis independent

\begin{tabular}{|l|}
\hline Summary/learning points \\
\hline - acute renal failure in pregnancy is still high in \\
India \\
- obstetric cortical necrosis has two peaks; in \\
early and late pregnancy \\
- the incidence of cortical necrosis is high in \\
post-abortal acute renal failure
\end{tabular}

Causes and clinical feature of renal cortical necrosis

- septic abortion

- haemolytic uraemic syndrome

- septicaemia with shock

- absolute anuria

- severe uraemic complications

- high mortality

failure in Indian patients. The high incidence of cortical necrosis in early pregnancy is a striking feature of this study as its occurrence is infrequent in early pregnancy in western countries. Thus, obstetric cortical necrosis had two peaks in our patients, one in post-abortum renal failure and the other in late pregnancy. The disease carries a high mortality, chiefly due to poverty and non-availability of chronic dialysis in this part of the country. The causes, clinical feature and learning points related to renal cortical necrosis are given in the boxes.

11 Levinsky NG, Alexander EA. Acute renal failure. In Brenner BM, Rector FC, (eds) The kidney. 2nd edn, Philadelphia: WB Saunder Company, 1981; pp L51-N1B1. 12 Gelfand MC, Friedman EA. Prognosis of renal allotransplants in patients with bilateral renal cortical necrosis Transplantation 1970; 10: 442-6.

13 American College of Surgeons/National Institute of Health. Transplant Registry, October 1976.

14 Effersoe P, Raaschou F, Thomanson AC. Bilateral renal cortical necrosis. A patient follow-up over 8 years. Am $\mathcal{f}$ Med
col 1962; 33: 455-8.

15 Rieselbach RE, Klahr S, Bricker NS. Diffuse bilateral cortical necrosis. A longitudinal study of the functional characteristics of residual nephrons. Am f Med 1967; 42 456-68.

16 Alwall N, Erlanson P, Tomberg, et al. Two cases of gross renal cortical necrosis in pregnancy with severe oliguria and anuria for 116 and 79 days, respectively. Acta Med Scand 1958; 161: 93 .

17 Moell H. Gross bilateral renal cortical necrosis during long periods of oliguria anuria. Roentgenologic observation in two cases. Acta Radial (Stockholm) 1957; 48: 355. 